\title{
Dust detector using piezoelectric lead zirconate titanate with current-to-voltage converting amplifier for functional advancement
}

\author{
Masanori Kobayashi ${ }^{1}$, Takashi Miyachi ${ }^{1}$, Maki Hattori $^{2}$, Seiji Sugita ${ }^{2}$, Seiji Takechi $^{3}$, and Nagaya Okada ${ }^{4}$ \\ ${ }^{1}$ Planetary Exploration Research Center, Chiba Institute of Technology, Narashino, Chiba 275-0016, Japan \\ ${ }^{2}$ Graduate School of Frontier Sciences, University of Tokyo, Kashiwa, Chiba 277-8582, Japan \\ ${ }^{3}$ Graduate School of Engineering, Osaka City University, Osaka, Osaka 558-8585, Japan \\ ${ }^{4}$ Honda Electronics Co., Ltd., Toyohashi, Aichi 411-3193, Japan
}

(Received January 6, 2012; Revised August 19, 2012; Accepted August 24, 2012; Online published March 12, 2013)

\begin{abstract}
This paper describes the concept of a dust monitor using lead zirconate titanate (PZT) ceramics with a large detection area. Its potential as a dust detector is experimentally demonstrated. The dust monitor has a small volume compared to an impact ionization detector with the same detection area, due to the PZT sensor. The PZT sensor, as a traditional device for the in-situ observation of hypervelocity dust particles, has been used for momentum measurement. The hypervelocity impact signals of PZT sensors are typically read by chargesensitive amplifiers. Instead, we suggest a new method that a current-to-voltage converting amplifier is useful for interpreting the impact signal of a PZT sensor arising from dust particles down to $0.5 \mu \mathrm{m}$ in radius. We propose that datasets of dust impacts can be obtained with a higher statistical accuracy, if the new method is applied to instruments on forthcoming interplanetary-space-cruising spacecrafts.
\end{abstract}

Key words: Dust monitor, piezoelectric sensor, PZT, current-to-voltage converting amplifier.

\section{Introduction}

Cosmic dust, in the range $10^{-18}-10^{-6} \mathrm{~g}$, is a basic component of space and has been directly observed by spaceborne missions in interplanetary space since the 1960s. Such dust particles have been identified as interplanetary dust particles (IPDs), $\beta$ meteoroids, interstellar dust (ISD), and dust ejected from the Jovian and Saturnian systems through in-situ observations by spacecraft between $0.3 \mathrm{AU}$ and $18 \mathrm{AU}$ heliocentric distances (Grün et al., 2001). The number of observed dust particles, however, has been statistically limited because of their low spatial density. Several models of dust flux in interplanetary space have been developed (Divine, 1993; Dikarev et al., 2005); however, these models can be improved by further observations with a higher statistical precision. Therefore, dust counters and analyzers with large detection areas have been recently proposed for a future space mission, DuneXpress (Grün et al., 2009). The payload of DuneXpress consists of seven sophisticated instruments for dust observation. This mission sheds light on many subjects that have remained because of insufficient statistical data from previous dust observations. The following parameters are some of the issues that will be addressed by DuneXpress:

- Size distribution of interstellar dust and the variation in flow direction and dispersion with particle size.

Copyright (C) The Society of Geomagnetism and Earth, Planetary and Space Sciences (SGEPSS); The Seismological Society of Japan; The Volcanological Society of Japan; The Geodetic Society of Japan; The Japanese Society for Planetary Sciences; TERRAPUB.
- Time variation in interstellar dust flows of various sizes.

- Ratio of cometary to asteroidal particles.

- Orbital characteristics of various types of cometary and asteroidal particles.

DuneXpress can determine dust trajectories with an accuracy of better than $3 \%$ in speed and $3^{\circ}$ in direction to distinguish interstellar dusts from interplanetary ones by their trajectories. Less-sophisticated instruments used in previous dust observation missions, however, have provided much insight into our understanding of dust populations in interplanetary space, although they could not determine accurate trajectories of detected dusts. For an example of those measurements, Grün et al. (1997) statistically discriminated between interstellar dust and interplanetary dust with a Dust Detector System having a sensitive area of $0.1 \mathrm{~m}^{2}$ and a wide field-of-view of $140^{\circ}$. Even though such observations cannot identify the population of individual dust particles, a statistical approach using the dataset can address the characteristics of the individual dust particle population, which are their size distribution, time variation, and orbital characteristics. For such a statistical approach, the number of detected dust particles should be as large as possible. It is an issue of forthcoming missions that the detection area should be enlarged. Consequently, ambiguities, based on statistical ambiguities, can be improved. In this paper, therefore, we discuss the idea that a number of segmented PZT sensors could cover a wide area of a spacecraft surface with smallscale resources. 
The current trend, concerning the detector area, is that current missions are equipped with dust detectors having a large detection area operated under minimal resources. The Arrayed Large-Area Dust Detector in Interplanetary Space (ALADDIN), onboard the engineering testing spacecraft Interplanetary Kite-craft Accelerated by Radiation Of the Sun (IKAROS) (Yano et al., 2011), uses two types of polyvinylidene fluoride (PVDF) sensors (9 and $20 \mu \mathrm{m}$ in thickness) and has a large total detection area of $0.54 \mathrm{~m}^{2}$. Of particular note is that ALADDIN requires a weight budget of only $247 \mathrm{~g}$ including its electronics, and the sensors are attached on the solar sail of IKAROS. This simple instrument provides the number of hypervelocity dust particles penetrating into the PVDF sheet with momentum larger than a particular threshold value. ALADDIN has detected more than 2000 events during one year of observation in heliocentric distances of 0.72-1.08 AU (Yano et al., 2011). The statistical accuracy of ALADDIN's observations is substantially better than that of previous observations. For example, HITEN MDC observed approximately 500 impacts of cosmic dust (effective detection area $100 \mathrm{~cm}^{2}$ ) during about three years at $1 \mathrm{AU}$ heliocentric distance (Auer, 2001), and NOZOMI MDC observed approximately 100 dust impacts (effective detection area $143 \mathrm{~cm}^{2}$ ) for about four years in heliocentric distances of 1.0-1.5 AU (Sasaki et al., 2007). ALADDIN's observations exhibited a trend similar to previous observations in which dust flux increased with decreasing distance from the Sun by an approximate factor of 10 (Yano et al., 2011). If ALADDIN continues to operate in interplanetary space for many years, it may observe solar modulation of interplanetary dust flux and dust particles in a cometary trail, which is yet to be observed. Despite its low functionality, such large-sensitive-area dust monitors could enhance the understanding of the dynamic behavior of dust particles in interplanetary space. In addition, observations through such missions as ALADDIN can provide insight into the previously-mentioned parameters. In this study, we have examined a dust-particle detector with a large detection area similar to that of ALADDIN. This study considered a small spacecraft such as HAYABUSA that cruised in interplanetary space for a lengthy period; thus, the dust instrument required fewer resources. For this purpose, in particular, a piezoelectric PZT sensor is incorporated in the instrument because its mechanical simplicity does not require much space even if it extends the detection area over the spacecraft surface. The PZT sensor has been widely accepted as a momentum sensor. However, momentum measurement alone is insufficient to uniquely determine the trajectory of dust particles. Thus, we draw attention to the potential of the PZT sensor to determine separately the mass and speed of dust particles, by taking a novel approach.

\section{Dust Detector Using Piezoelectric PZT}

Piezoelectric crystals (PZCs) have been widely used as supersonic transducers and impact sensors for the in-situ observation of cosmic dust. The response of a PZC sensor is related to the momentum of a dust particle at low speed $(v<1 \mathrm{~km} / \mathrm{s})$ and includes the term $v^{2}$ due to the recoil from impact ejecta. In practice, the contribution from the recoil is negligible in comparison with the measurement precision (Auer, 2001). The advantages of PZC sensors are mechanical simplicity and stiffness, unnecessity of a required bias voltage, and high temperature and radiation tolerance. Thus, PZC sensors have been used for in-situ dust observations. Thick PZC and thin (typically $0.1 \mathrm{~mm}$ ) piezoelectric diaphragms were employed on spacecraft in the early 1970s (Auer, 2001). As part of the Dust Impact Detection System (DIDSY), several PZCs were attached to the dust shields of the Giotto spacecraft launched to study the comet Halley (McDonnel et al., 1986). The Mercury dust monitor (MDM) will be onboard the BepiColombo/Mercury magnetosphere orbiter (MMO) to be launched in 2014 (Nogami et al., 2010). MDM was proposed to investigate the dust environment around Mercury. Hence, PZC sensors constructed of PZT have been adopted because of their high temperature and radiation tolerance, in addition to a high piezoelectric constant. Four square plates of PZT, each $40 \mathrm{~mm} \times 40 \mathrm{~mm} \times 2 \mathrm{~mm}$, will be installed on a side panel of the MMO. The PZT sensors can easily detect vibrations from origins other than impacts on the detection area. Concerning true-false discrimination, therefore, it is necessary to examine the waveforms of the signals read by an amplifier. For the BepiColombo/MDM, the signal waveform from the sensors will be digitalized by a flash analog-todigital converter (ADC), with a sampling rate of $20 \mathrm{MHz}$, in onboard electronics. Because true dust impact events, and fake events that are likely to be generated by dust impact in ambient instruments or by thermal strain, are not distinguished onboard, the waveform will be downlinked to the ground. In general, severe limitations exist in available resources such as power consumption and communication rate in space mission; therefore, power consumption and telemetry for recording and downloading the waveform should be conserved. This is especially critical if a number of signal channels need to be read as a result of numerous PZT sensors used to enlarge sensitive areas for cosmic dust observation. In previous studies of piezoelectric dust sensors, responses of the sensor have been measured in charge or voltage by charge-sensitive or voltage amplifiers for observing momentum transfer during the impact. Here, we suggest that the signals from the PZT sensor should be read in a current mode by the amplifier, which would enhance the function of the PZT sensor. In fact, the determination of momentum, size, and speed for hypervelocity microparticles, and true-false discrimination, may be facilitated.

\section{Signal Readout of the PZT Sensor for a Hyper- velocity Microparticle}

\subsection{Signal generation of dust impact}

In this section, we briefly describe stress generation during collision impact. For simplicity, we cite the problem of elastic waves generated by cylindrical-bar collisions that typically appears in dynamics textbooks, such as that written by Meyers (1994). When a cylindrical projectile with a length $L$ collides with a cylindrical bar, a rectangular pulse of length $2 L$ propagating through the bar is generated during a time interval of $2 L / C$, if the bar and projectile are of the same materials. The stress generated by the impact $\sigma$ at a speed $V$ is given by $\sigma=\rho C U_{p}=1 / 2 \rho C V$, where $\rho$, 
$C$, and $U_{p}$ are the density, longitudinal speed, and particle speed of the target material, respectively. Accordingly, we can theoretically determine the size and speed of the projectile from the pulse shape of the generated stress. In reality, however, the projectile and the target have more complicated structures and are composed of different materials. Furthermore, hypervelocity impact causes an inelastic collision, generating shock waves and can evaporate some portion of the materials; therefore, impact duration is not a simple function of the size of the projectile particle (Melosh, 1989). Nevertheless, the pulse shape of the stress can provide an insight for predicting projectile parameters by using such an empirical law derived from dust acceleration experiments, as shown in figure 5 of Weishaupt (1987), in which the signal rise time depends on the projectile velocity and size. If a dust particle collides with the surface perpendicular to the thickness direction of a PZT sensor plate and parallel to the polarization direction, the generated longitudinal stress wave along the polarization direction can be electrically observed. The time variation of charge signals converted from the stress in the PZT target corresponds to the stress wave. The stress wave can be correctly read by a charge sensitive amplifier (CSA) with a response speed faster than the time variation. Weishaupt (1987) used a CSA to read impact signals of various-sized glass beads hitting a PZT sensor and demonstrated experimentally that the rise time of the charge signals was related to the sizes of the glass bead projectiles. The rise time corresponds to the time interval in which stress increases during the impact. When a projectile particle collides with a target PZT sensor, the mechanical strain due to the generated impact pressure is converted to electric charges appearing on the surface of the sensor that increases as the strain becomes greater. That is, the electric charge generated by the impact is a function of the elapsed time of the collision between the projectile and the target. The charge generation due to dust particle impact is extremely small, and PZT sensors have large electric capacities because the piezoelectric element has a dielectric constant, $\varepsilon=1500$. Hence, the signals of PZT sensors are usually read with a CSA.

\subsection{Signal readout of the PZT sensor with a current-} to-voltage converting amplifier

A CSA is essentially composed of an operation amplifier and a feedback capacitor. When a CSA matches the electrostatic capacitance of the sensor, it can read tiny signals even if the sensor's electrostatic capacitance varies because of factors such as temperature dependence. In addition, a CSA generally has a frequency range up to approximately $10 \mathrm{MHz}$, because the amplification of signals in higher frequencies creates an instability resulting in oscillation noise. Hence, a CSA does not read the signal of the impact stress, which is an extremely short timescale phenomenon. Instead, it reads stationary wave signals with the resonance frequency determined by the sonic speed and the thickness of the PZT sensor. An example is $1.1 \mathrm{MHz}$ for a PZT sensor with a longitudinal wave speed of $4.4 \mathrm{~km} / \mathrm{s}$ and a thickness of $2 \mathrm{~mm}$. The magnitudes of signals consisting of resonance frequency and harmonic resonance components are proportional to the momentum transferred from the incident particles to the target sensor. By reading the signal during

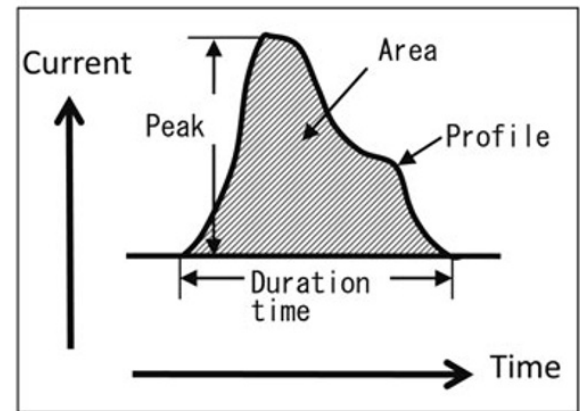

(a) Measurements

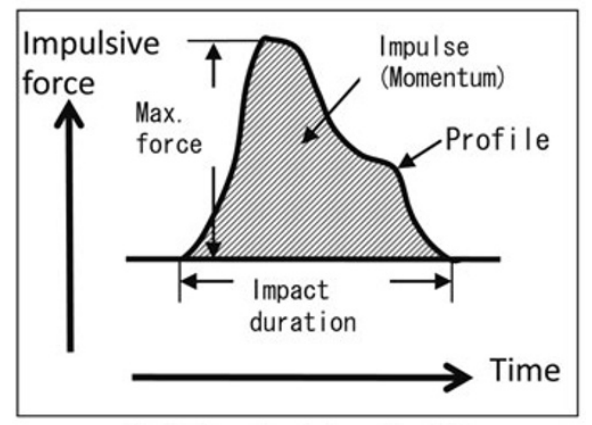

(b) Physical implications

Fig. 1. Correspondence of current signal measurements to physical implications.

the dust impact, we may determine the mass (as predicted from the size, assuming the mass density) and speed of dust particles separately. As previously mentioned, a CSA is not appropriate for fast signal readouts of small dust particles of a few $\mu \mathrm{m}$ or less, because the impact duration is approximately $1 \mathrm{~ns}$. Instead, an amplifier known as a current-tovoltage converting amplifier (CVA) is employed to read the signal from a sensor in a current mode and output the amplified signal as a voltage. A CVA should have a low input impedance compared with the impedance of the sensor, which, in this case, is a capacitative reactance. Therefore, the electric charge generated in the sensor will flow into the CVA as an electric current. An advantage of a CVA is that the frequency range can be extended to frequencies as high as GHz. In addition, its rated input load capacitance is high, similar to that observed in CSAs. For example, a CVA that was fabricated for an experimental demonstration, as mentioned in the following section, can accept an input load capacitance up to $100 \mathrm{nF}$, equivalent to the capacitance of a PZT sensor plate of $120 \mathrm{~mm} \times 120 \mathrm{~mm} \times$ $2 \mathrm{~mm}$. Here, we briefly consider the physical implications of electric current measurements on a PZT sensor. Figure 1 shows a correspondence between the measurements of current signal and its physical implications. Essentially, the electric current is the time derivative of an electric charge. The magnitude of an electric charge on the PZT sensor represents the momentum of the projectile particle, while the pulse height of the current signal represents the impulsive force during the collision of the projectile and target. The area found by the integral of the electric current pulse over the time duration corresponds to the impulse of the projectile dust particle, which is approximately proportional to the 
Table 1. Correspondence of measurements to quantities of impact phenomena and physical properties of projectile dust particles.

\begin{tabular}{|c|c|c|}
\hline Measurements & Quantities of impact phenomena & Quantities of projectile dust particles \\
\hline \hline Magnitude of current & Impulsive force & Impact speed, size, and mass \\
\hline Duration time & Impact duration & Size and longitudinal wave speed \\
\hline Area of current pulse & Impulse & Momentum \\
\hline
\end{tabular}

projectile momentum. Table 1 shows the correspondence of the measurements to the physical quantities of the impact phenomena of the projectile dust particles during impact and the related physical quantities of the projectile particle. When a projectile microparticle collides with a PZT sensor, the PZT sensor generates an electric charge, the total amount of which is proportional to the momentum transfer (or impulse) from the microparticle. The electric charge has a time variation that corresponds to the time derivative of the impulse, namely the impulsive force exerted on the sensor. The impulsive force is an integral of the collisional stress, $\sigma$, with regard to the contact area of collision, $\sim L^{2}$. The duration time of the collision is $\sim 2 L / C$ as described in the previous section. Although the functions of these parameters are not simple to acquire, we can obtain additional physical properties of the projectile dust particle from the current amplifier output signals. This method gives an extent of measuring the accuracy of the particle mass. The propagation speed of the longitudinal stress waves (or acoustic waves) depends on the material. The conversion of the duration time of the CVA output signal into the size of the microparticle is calculated with the acoustic wave speed. The variation of the acoustic wave speed of natural materials that ranges from 3 to $6 \mathrm{~km} / \mathrm{s}$ causes a measurement error of the microparticle size. Besides, the mass of the microparticles can be calculated from the size assuming a spherical particle and a certain value of material density. This causes a measurement error of the microparticle mass. At present, it is difficult to exactly estimate the accuracies of the speed measurement and the density estimate. Considering the values above, we consider that the mass will be determined within a factor of one. The boundary of the error will be studied with accelerators in the future. We have designed a CVA prototype for reading current signals generated during impact on a piezoelectric PZT sensor, and have conducted experiments to evaluate its performance. Experimental details and results are described in the following section.

\section{Experiments on the Current Signal Readout of Impact on a Lead Zirconate Titanate Sensor}

We used an active Q-switch YAG pulse laser unit (MINILASE II-10; New Wave Research Inc., California) with a 1064-nm wavelength, 6-8-ns pulse duration, and a $<30$ $\mathrm{mJ}$ per pulse to simulate impact on the surface of a PZT sensor through light pressure. The PZT sensor (Honda Electronics Co., Ltd., Japan) was the same product as that installed in the BepiColombo/Mercury Dust Monitor (Nogami et al., 2010). A PZT sensor with a detection area of $8 \mathrm{~mm} \times 8 \mathrm{~mm}$, a thickness of $8 \mathrm{~mm}$, and thin layers of silver electrode with thicknesses of $5 \mu \mathrm{m}$ was attached on each side of the unit. The electric capacitance was $0.23 \mathrm{nF}$. A laser spot was focused to a diameter of approximately $1 \mathrm{~mm}$ on the sensor surface. Although impact generation by light pressure of a pulse laser (not ablation pressure) is not the same as that arising from the collision of materials, this type of impact is adequate for investigating the signal response of a PZT sensor read by a CVA. For example, a laser pulse of $30 \mathrm{~mJ}$ with a spot of $1 \mathrm{~mm}$ in diameter generated an impact pressure of approximately $14 \mathrm{mN}$ or higher, depending on the reflectivity of the surface.

We fabricated a CVA with a response time of approximately $6 \mathrm{~ns}$ and input impedance of $20 \Omega$. Although this CVA was fabricated simply for our experiment, the response time was much faster than those used in previous studies by a factor of about 10 . Laser pulses irradiated through the thickness direction of the PZT sensor generated light pressure on the PZT sensor. The output waveforms of the CVA were recorded by a digital oscilloscope (WaveMaster 806Zi, LeCroy Corporation, New York) with capability of $6 \mathrm{GHz}$ and $40 \mathrm{GS} / \mathrm{s}$. Figure 2 shows a typical waveform of the current signal of the laser shot on the PZT sensor; Fig. 2(b) is a magnified version of Fig. 2(a) in time scale. In these figures, the vertical axes show an output voltage signal of the CVA corresponding to the current flowing to the CVA. The positive output signals show compression, and the negative signals show rarefaction in the thickness direction of the PZT sensor. As shown in Fig. 2(a), the waveform has periodically-appearing multiple pulses. Figure 2(b) shows that the first pulse has a positive peak and a negative peak. Two vertical solid lines in the figure depict that the positive peak (1) (compression) was definitely generated at the time of the laser pulse impact, and that the negative peak (2) (rarefaction) could be generated by restoration. After approximately $2 \mu \mathrm{s}$, corresponding to the propagation time of the longitudinal wave along a thickness direction of $8 \mathrm{~mm}$, the next sharp pulse appeared. However, the phase was inverted, and the amplitude was attenuated. Subsequent pulses appeared in approximately $2-\mu$ s intervals with a phase inversion. The waveform of the hypervelocity impact read by the CVA consists of discrete wave packets and the first pulse clearly starts with a positive peak due to the compression of the sensor material by collision. On the other hand, one read by a CSA (for example, see Miyachi et al. (2005)) has a continuous waveform with a period determined by the sensor thickness and the acoustic wave speed. As mentioned in the previous section, a current signal waveform is in the form of the time derivative of a charge signal waveform. The first pulse had a rapid rise time for the leading edge, which slowed in subsequent pulses. It can be considered that a stress wave 

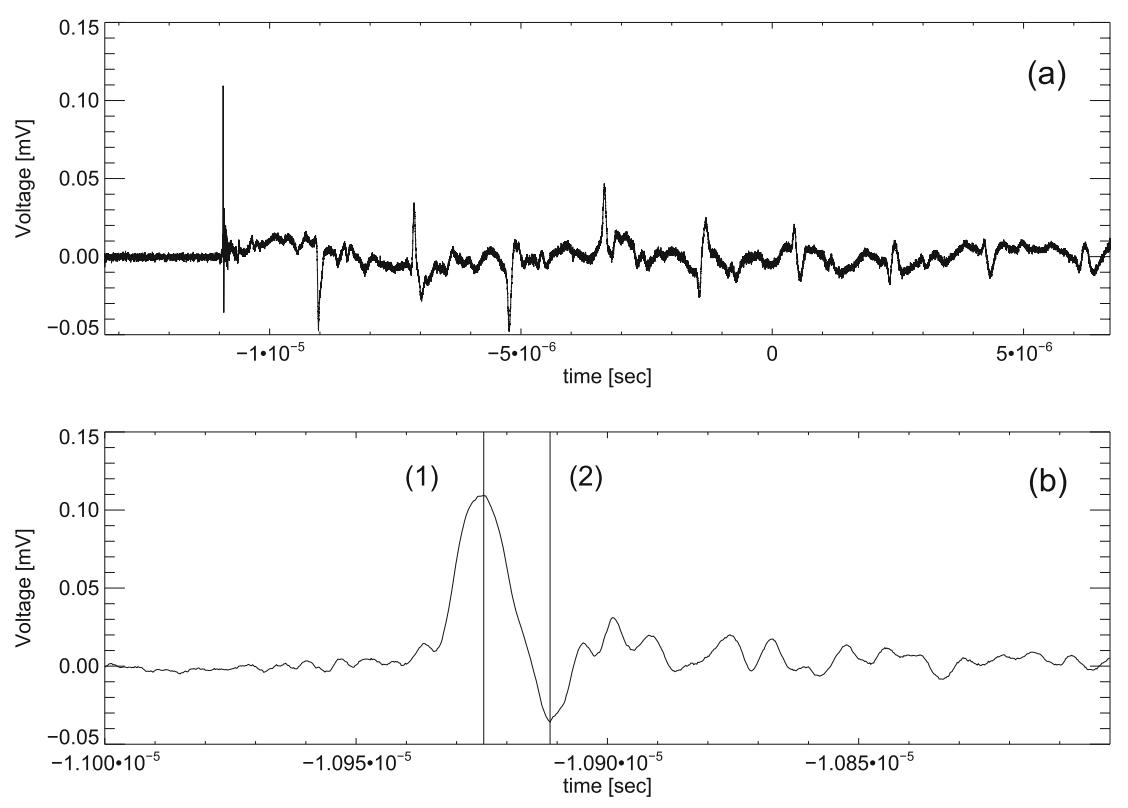

Fig. 2. Example of a typical waveform of the current signal of a $25.9 \mathrm{~mJ}$ laser pulse shot on the PZT sensor in a long timescale of $20 \mu \mathrm{s}$ (a), and in the magnified time scale (b) for the first pulse.

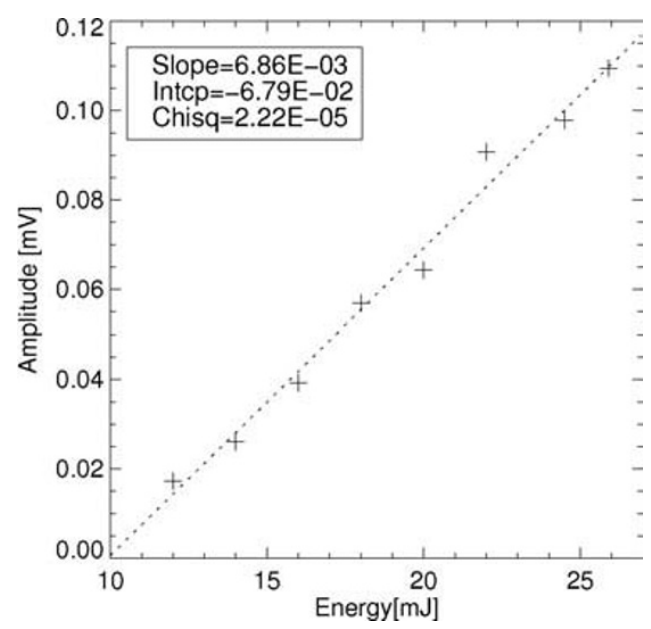

Fig. 3. Linear relationship between the amplitudes of the first pulses of the signal waveforms and the laser pulse energies. The slope, intercept and reduced chi square value of the linear curve fitting are shown.

was propagated through the PZT sensor to the other surface and was reflected at the free end with dispersion. Although only small variations were apparent between pulses in the waveform in Fig. 2(a), the electric charge indeed appeared on the surface of the PZT sensor. Figure 3 shows a linear relationship between the amplitudes of the first pulses of the signal waveforms and the laser pulse energies; however, several data points deviated from the linear curve because of the instability of the laser shot. The negative intercept of the plots shown in Fig. 3 is due to the absorption of laser energy at the optical focal lens right before the target sensor. In this case, the time profile of the laser pulse shot, which was approximately a rectangular pulse of about $7 \mathrm{~ns}$ in duration, could not be determined because the response speed of the CVA was not sufficiently fast. As shown in Fig. 3, the first-pulse amplitude is related to the impulsive force produced by the laser shot. Impact by laser shot, however, differs technically from that by material collisions. In addition, the CVA used in this study does not have sufficient performance to measure a $1-\mu \mathrm{m}$-size microparticle, but only for $10-\mu \mathrm{m}$-size, or larger, microparticles. We feel that the result above is promising enough to develop a new method which can measure the size and speed of a hypervelocity microparticle using the PZT sensor. For further studies, we will experimentally demonstrate this new dust monitor in terms of the measurement of a $1-\mu \mathrm{m}$-scale microparticle, after an improvement of the response speed of the CVA. In this study, our final goal is to detect impact phenomena arising from microparticles with sizes of approximately $1 \mu \mathrm{m}$ and speeds higher than $1 \mathrm{~km} / \mathrm{s}$. In this case, the duration time of collision corresponds to the propagation time of the compression stress wave generated at the first collision point through to the opposite side where the wave is reflected to be a rarefaction wave traveling back to the first point. The timescale of such detection target phenomena is approximately $1 \mathrm{~ns}$. Also, the size of the 

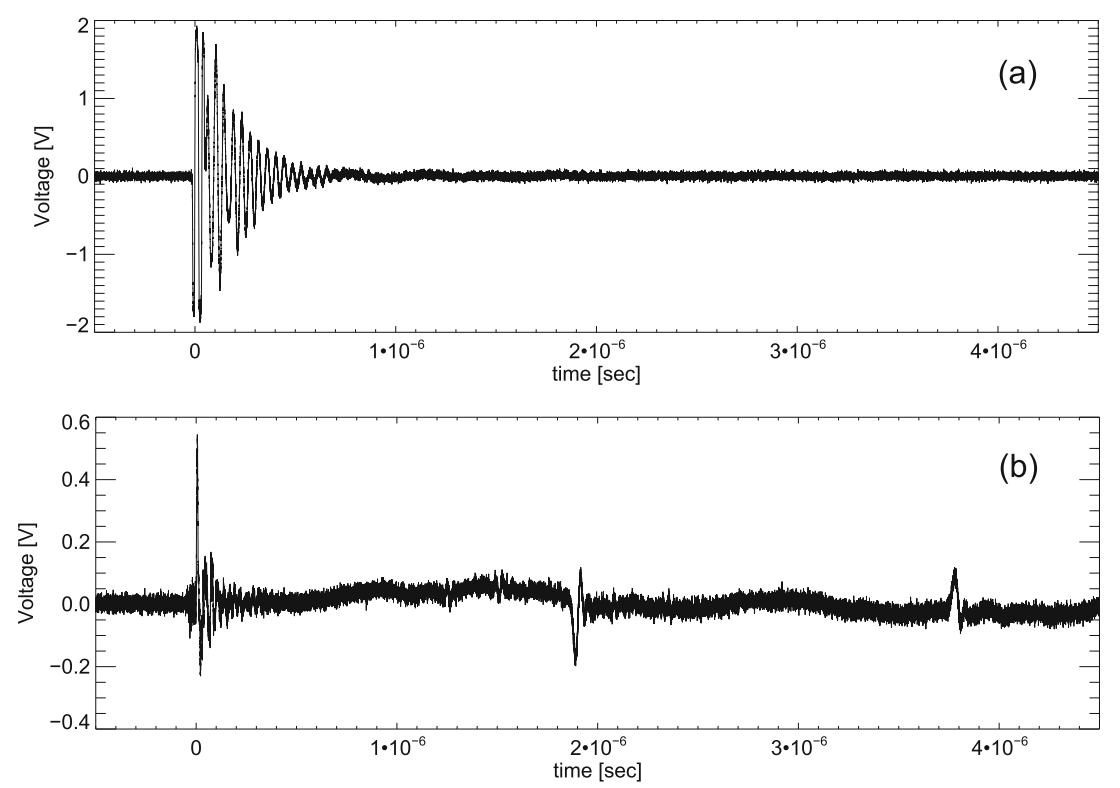

Fig. 4. Comparison of (a) output signals of the PZT sensor by finger-snapping on its frame (false event) and (b) a typical true event in which an impulsive force is applied to the sensor plane (true event).

impact phenomena corresponds to the area where the impulsive force is exerted: approximately the cross-sectional size of the projectile microparticle. In addition, we utilized laser radiation pressure to apply an impulsive force to the sensor in order to avoid ablation damage of the sensor surface. For that reason, the spot size of the laser was set to be $1 \mathrm{~mm} \phi$ and the area over which the impulsive force was exerted was also of the same space scale. The duration time of the impulsive force was about $7 \mathrm{~ns}$, which was the same scale as the laser pulse duration. As described above, the impulsive forces generated by pulse laser irradiation in this experiment differed in timescale and space scale from our target phenomena of microparticle collision. The difference in space scale, however, does not affect the charge signal generated by the piezoelectric effect. With a laser pulse of $30 \mathrm{~mJ}$ and a spot size of $1 \mathrm{~mm} \phi$, and a duration time of $7 \mathrm{~ns}$, the generated impulsive force is approximately $14 \mathrm{mN}$ and the generated impulse is about $100 \mathrm{pg} \mathrm{km} / \mathrm{s}$. The duration time of $7 \mathrm{~ns}$ corresponds to that of an impulsive force generated by the collision of a microparticle of a size $10 \mu \mathrm{m}$ with a target, supposing the propagation speed of the stress wave (or acoustic wave) in the projectile material is about $4 \mathrm{~km} / \mathrm{s}$ : the mass of the microparticle is about $100 \mathrm{pg}$ assuming a mass density of $2.5 \mathrm{~g} / \mathrm{cc}$. In other words, this laser experiment simulated a microparticle with a mass of $100 \mathrm{pg}$ and a speed of $1 \mathrm{~km} / \mathrm{s}$ colliding with a target PZT sensor. The mass of $100 \mathrm{pg}$ is much larger than that of our targeted microparticle, however, the duration time is appropriate to evaluate the performance of the prototype CVA, and the feasibility of the new measurement method has been demonstrated.

\subsection{Pulse shape discrimination}

The output signal waveform of the PZT sensor with a CVA is clearly defined, as shown in Fig. 2, and determines a true-false discrimination of the sensor output signal. A piezoelectric sensor, such as a PZT sensor, is sensitive to mechanical vibration, including that from external noise sources such as thermal strain in the mechanical support. Accordingly, the signal waveform should be processed by flash ADC (FADC) and downlinked to the ground for a truefalse analysis, as previously mentioned in relation to BepiColombo MDM. Figure 4 compares the output signals of the PZT sensor by flicking the sensor frame (a) and a typical true event in which the impulsive force is applied to the sensor plane (b). The criterion of signal-noise discrimination is sufficiently simple to enable a waveform to be identified as a signal or a noise. Namely, the true-event waveform consists of separate peaked waves and must start with a positive peak due to the compression of the sensor material by collision. The separate peaks appear every $2 \mu$ s with an occurring phase inversion, because the longitudinal stress wave propagates through the thickness direction with a speed of about $4 \mathrm{~km} / \mathrm{s}$ at both free end surfaces. In contrast, the noise event has a continuous waveform and the amplitude attenuates rapidly. Thus, true-false discrimination can be simply conducted onboard. Because of that, FADC is not necessary to read out the waveform of the PZT sensor signal output and, accordingly, large amounts of power consumption and data transmission of FADC can be avoided.

\section{Conceptual Design of a Large-area Dust Detec- tor Using a PZT Sensor with CVA}

As mentioned in the previous section, we have demonstrated the possibility of a PZT sensor with a CVA within a specific range. From the perspective of practical use, the response speed of the CVA should be faster; therefore, digital electronics to measure the time duration of the pulse signal of the stress wave should be considered. Ultra-high-speed comparators are commercially available, such as the ADCMP582 manufactured by Analog Devices, which has an equivalent input rise time bandwidth of $8 \mathrm{GHz}$. Thus, the time duration of the pulse signal can be measured with a precision of $0.5 \mathrm{~ns}$, even in space. Considering elastic approximation, a dust particle with a radius of approximately 
$0.5 \mu \mathrm{m}$ generates a pulse duration of approximately $0.5 \mathrm{~ns}$ in current signal. From the perspective of hardware design, the lower limit of dust size measured by a PZT sensor with a CVA can be $0.5 \mu \mathrm{m}$. According to Grün et al. (1985), the flux of interplanetary dust greater than $0.5 \mu \mathrm{m}$ at 1 AU heliocentric distance is approximately $10^{-4} \mathrm{~m}^{-1} \mathrm{~s}^{-1}$; therefore, it is expected that approximately 1700 dust impacts would be obtained by a sensor with a detector area of $0.54 \mathrm{~m}^{2}$, which is the same area as that of ALADDIN. The detection area of a PZT sensor is limited by the input load capacitance of the amplifier for signal readout. Given a rated input load capacitance of $100 \mathrm{nF}$ is affordable for a CVA, the CVA can read out the signal output from a PZT sensor with dimensions of $120 \mathrm{~mm} \times 120 \mathrm{~mm} \times 2 \mathrm{~mm}$. In such a case, approximately 37 plates of PZT sensors are necessary to cover $0.54 \mathrm{~m}^{2}$. Thus, the larger the detection area of each PZT sensor, the smaller the number of PZT sensors. We have considered the resource requirement of this new method in comparison with other methods. An impact ionization detector is an instrument to measure the masses and the speeds of microparticles, as well as a PZT sensor with a CVA. As a part of the DuneXpress mission, an impact ionization detector with a large detection area, called Dust Camera 3 or DC3, was proposed. Actually, DC3 will be used with a trajectory detector of DuneXpress, DT1, but an impact ionization detector such as DC3 can obtain the masses and the speeds of hypervelocity microparticles by itself. DC3 comprises 25 sensing modules, each $9 \times 9 \mathrm{~cm}^{2}$, mounted in a $5 \times 5$ array, thereby it has a detection area of $0.2 \mathrm{~m}^{2}$, a weight of $9 \mathrm{~kg}$, a volume of $50 \times 50 \times 23 \mathrm{~cm}^{3}$, and a power consumption of $9 \mathrm{~W}$ (Grün et al., 2009). On the other hand, drawing on the practical design of BepiColombo MDM (Nogami et al., 2010), the dust monitor using the new method with the same detection area of $0.2 \mathrm{~m}^{2}$, will require less resources, a weight of $8 \mathrm{~kg}$, a power consumption of $6 \mathrm{~W}$ and a volume of $50 \times 50 \times 1 \mathrm{~cm}^{3}$ : the occupied volume is significantly reduced.

\section{Summary and Future Plans}

We have studied the potential of a new method of dust monitoring having a large detection area but small volume. In this study, a PZT sensor with a CVA is studied and we used pulse laser shots to generate impulsive forces in order to simulate hypervelocity microparticle impacts on the sensor. The signal readout of the CVA showed a linear response to the impulsive force on the PZT sensor. In addition, the signal readouts exhibited distinctive waveforms, that can be identified by onboard simple-logic electronics, enabling pulse discrimination from false impact events, such as that on the peripheral part of the sensor or by thermal strain. Our goal is to examine dust particles with sizes of $0.5 \mu \mathrm{m}$ in radius so that the CVA can be improved to be faster. In addition, the following circuit design will require a high-speed comparator with a capability to process signals with a time precision of $0.5 \mathrm{~ns}$ or less. Existing technology is sufficient to create the instrumentation for this conceptual design. We will fabricate a CVA using a printed circuit board for faster response and vacuum. In future research, we will conduct a dust acceleration experiment using a PZT sensor with a CVA, light gas guns, and electrostatic accelerators.
Acknowledgments. We would like to give heartfelt thanks to Mr. Ohwada, and Mr. Shinkawa from Kaizu Works Corporation who provided us fruitful advice on our current-to-voltage converting amplifier. We also gratefully appreciate the generosity of Prof. T. Kawamura and Prof. K. Nogami from Dokkyo Medical University that provided us with a pulse laser in our experiments. Special thanks also go to Mr. Terry Byers from NASA Johnson Space Center and an anonymous reviewer whose opinions and information have helped us very much throughout the production of this paper.

\section{References}

Auer, S., Instrumentation, in Interplanetary Dust, edited by Grün et al., 804 pp, Springer-Verlag, Berlin Heidelberg New York, 2001.

Dikarev, V., E. Grün, J. Baggaley, D. Galligan, M. Landgraf, and R. Jehn, The new ESA meteoroid model, Adv. Space Res., 35, 1282-1289, 2005.

Divine, N., Five populations of interplanetary meteoroids, J. Geophys. Res., 98, 17029-17048, 1993.

Grün, E., H. A. Zook, H. Fechtig, and R. H. Giese, Collisional balance of the meteoritic complex, Icarus, 62, 244-272, 1985.

Grün, E., P. Staubach, M. Baguhl, D. P. Hamilton, H. A. Zook, S. Dermott, B. A. Gustafson, H. Fechtig, J. Kissel, D. Linkert, G. Linkert, R. Srama, M. S. Hanner, C. Polanskey, M. Horanyi, B. A. Lindblad, I. Mann, J. A. M. McDonnell, G. E. Morfill, and G. Schwehm, South-north and radial traverses through the interplanetary dust cloud, Icarus, 129, 270-288, 1997.

Grün, E., M. Baguhl, H. Svedhem, and H. A. Zook, In situ measurement of cosmic dust, in Interplanetary Dust, edited by Grün et al., 804 pp, Springer-Verlag, Berlin Heidelberg New York, 2001.

Grün, E., R. Srama, N. Altobelli, K. Altwegg, J. Carpenter, L. Colangeli, K.-H. Glassmeier, S. Helfert, H. Henkel, M. Horányi, A. Jäckel, S. Kempf, M. Landgraf, N. McBride, G. Moragas-Klostermeyer, P. Palumbo, H. Scholten, A. Srowig, Z. Sternovsky, and X. Vo, DuneXpress, Exper. Astron., 23(3), 981-999, 2009.

McDonnell, J. A. M., W. M. Alexander, W. M. Burton, E. Bussoletti, D. H. Clark, G. C. Evans, S. T. Evans, J. G. Firth, R. J. L. Grard, E. Grün, M. S. Hanner, D. W. Hughes, E. Igenbergs, H. Kuczera, B. A. Lindblad, J.-C. Mandeville, A. Minafra, D. Reading, A. Ridgeley, G. H. Schwehm, T. J. Stevenson, Z. Sekanina, R. F. Turner, M. K. Wallis, and J. C. Zarnecki, The Giotto dust impact detection system, ESA Special. Publication, ESA SP-1077, 85-107, 1986.

Melosh, H. J., Impact cratering: a geologic process, in Oxford Monographs on Geology and Geophysics, 11, 245 pp, Oxford University Press, New York, 1989.

Meyers, M. A., Dynamic Behavior of Materials, 668 pp, John Wiley \& Sons, Inc., New York, 1994.

Miyachi, T., M. Fujii, N. Hasebe, M. Kobayashi, G. Kuraza, A. Nagashima, Y. Nakamura, O. Okudaira, N. Yamashita, K. Nogami, T. Iwai, S. Sasaki, H. Ohashi, S. Hasegawa, H. Yano, H. Shibata, N. Okada, and T. Tou, Response from piezoelectric elements appearing immediately after collisions with silver particles, J. Appl. Phys., 98, 014110-1-7, 2005.

Nogami, K., M. Fujii, H. Ohashi, T. Miyachi, S. Sasaki, S. Hasegawa, H. Yano, H. Shibata, T. Iwai, S. Minami, S. Takechi, E. Grün, and R. Srama, Development of the Mercury dust monitor (MDM) onboard the BepiColombo mission, Planet. Space Sci., 58, 108-115, 2010.

Sasaki, S., E. Igenbergs, H. Ohashi, R. Senger, R. Münzenmayer, W. Naumann, E. Grün, K. Nogami, I. Mann, and H. Svedhem, Summary of interplanetary and interstellar dust observation by Mars Dust Counter on board NOZOMI, Adv. Space Res., 39, 485-488, 2007.

Weishaupt, U., Hypervelocity impact of small masses on large surfaces of piezoelectric ceramics, Int. J. Impact Eng., 5, 663-670, 1987.

Yano, H., T. Hirai, C. Okamoto, N. Ogawao, M. Tanaka, and The IKAROSALADDIN Team, In-situ dust flux measurement inside 1 AU heliocentric distance by the IKAROS solar sail spacecraft, 8th Annual Meeting AOGS 2011, Taipei, August 8 to 12, 2011, Article number PS02-A047, 2011.

M. Kobayashi (e-mail: kobayashi.masanori@perc.it-chiba.ac.jp), T. Miyachi, M. Hattori, S. Sugita, S. Takechi, and N. Okada 